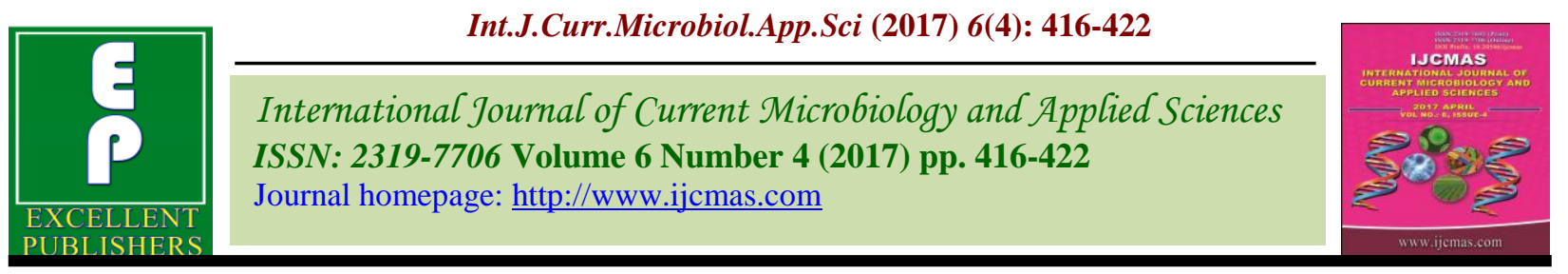

Original Research Article

https://doi.org/10.20546/ijcmas.2017.604.047

\title{
Time Related Emergence of Bacterial Pathogens and their Antibiograms in Burn Wound Infections in a Tertiary Care Hospital
}

\author{
Shashi S. Sudhan ${ }^{1 *}$, Preeti Sharma ${ }^{1}$, Kunal Sharma ${ }^{2}$, \\ Monika Sharma ${ }^{1}$ and Sorabh Singh Sambyal ${ }^{1}$ \\ ${ }^{1}$ Department of Microbiology, Govt. Medical College and Hospital Jammu, India \\ ${ }^{2}$ Department of Surgery, Govt. Medical College and Hospital Jammu, India \\ *Corresponding author
}

\section{A B S T R A C T}

\section{Keywords}

Burn injury, $S$. aureus, Screening, Antibiograms. Pseudomonas sp.

Article Info

\section{Accepted:}

02 March 2017

Available Online: 10 April 2017
Burn injury is a life-threatening event associated with both high morbidity and mortality. Burnarea provides a suitable site for bacterial multiplication and also becomes a more persistent richer source of infection, mainly because of the longer duration of patient stay in the hospital. The survival rates for burn patients have however improved substantially in the past few decades due to advances in modern medical care in specialized burn centers. The present study was undertaken to provide an insight to evaluate time related changes in microbial flora and their antibiotic susceptibility pattern occurring in the burn unit of Government Medical College and Hospital, Jammu from January 2013 to June 2013. The specimens were processed according to standard laboratory protocols, isolates were identified by conventional biochemical methods and antimicrobial susceptibility was performed by Kirby-Bauer disc diffusion method. A total of 63 patients were enrolled in the present study. Among these, 49(77.77\%) (Showed evidence of burn wound infection whereas 14 (22.22\%) (had no evidence of infection. Pseudomonas aeruginosa was the commonest pathogen isolated (27\%) followed by Klebsiella sp. (26\%), S. aureus (17\%), Proteus sp. (11\%), Streptococcus sp. (10\%), Enterococcus sp. and Enterobacter sp. (4\%) respectively and Acinetobacter sp (1\%). Gram-positive bacteria showed absolute resistance to Pencillin and absolute sensitivity to Vancomycin whereas in Gram negative bacteria $100 \%$ resistance to Ampicillin and $85.18 \%$ sensitivity to Piperacillin-Tazobactum was observed. There was a transition of bacterial growth from Gram-positive (Staphylococcus aureus being the most common) during the first week to Gram-negative (Pseudomonas species being the most common) in the subsequent weeks of stay. Gram positive bacteria and Gram negative bacteria were found sensitive to Vancomycin and Piperacillin-tazobactum.

\section{Introduction}

Infection in burn wound is still considered the most important cause of morbidity and mortality in all ages and in both developed and developing countries (American Burn Association. 2000). Despite considerable advances in the overall management of burn injuries, infection and the resultant sepsis continues to be a formidable foe for burn care providers. Approximately 50-75\% of mortality amongst burn patients after the initial resuscitation phase, is attributable to various infectious complications (Lionelli et 
al., 2005; Atiyeh et al., 2005). Burn injury patients are at high risk of infections for a variety of reasons like the readily available exposed body surface, immunocompromizing effects of burns, invasive diagnostic and therapeutic procedures and prolonged hospital stay. Patient factors such as age, extent of injury, and depth of burns with microbial factors such as the type and number, enzyme/toxin production and motility of organisms are the determinants of invasive infection. Superficial bacterial contamination of the wound can easily advance to invasive infection in these patients (Baker et al., 1979). The degree of bacterial wound contamination has a direct correlation with the risk of sepsis. Factors that are associated with improved outcome of burn injury patients and prevention of infections among them predominantly include early excisions and grafting of deep burns together with aggressive infection-control measures (Apelgren et al., 2002). These changes potentially lead to the emergence of antibiotics resistant isolates and treatment failure (Lemmen et al., 2004). Sources of organisms are found in the patient's own endogenous (normal) flora, from exogenous sources in the environment, and from healthcare personnel (Pruitt et al., 1998). Survival in burn patients has improved tremendously with the Gram-positive bacteria that survive the thermal insult and heavily colonize the wound surface within the first 48 hours unless topical antimicrobial agents are used (Zorgani et al., 2002). These wounds are subsequently colonized with other microbes such as Gram-negative bacteria and yeasts derived from the host's normal gastrointestinal and upper respiratory flora and/or from the hospital environment or that are transferred via a health care worker's hands (Karyoute, 1989).

Over the last several decades, Gram-negative organisms have emerged as the most common etiologic agents of invasive infection because of their large repertoire of virulence factors and antimicrobial resistance (Challa). Various antibiotics are used in the treatment of burn sepsis. The efficacy of various topical antimicrobials in common use in modern burn centers is dynamic due to the ability of microorganisms to develop resistance rapidly. The sustained potency of individual agents depends on the extent of use and the resident nosocomial flora within any specialized burn center (Neelam et al., 2004). In order to accurately detect and track emerging trends in topical antimicrobial resistance in modern burn units, it is essential that standard reproducible methods be published for clinical implementation. The present study aims to study the microbial profile of burn wound infections in burn patients, time related changes and to evaluate the antibiotic sensitivity of causative agents.

\section{Materials and Methods}

This prospective study of burn wound infections was conducted over a period of six months from January 2013 to June 2013 at Government Medical College and Hospital $(\mathrm{GMCH})$, Jammu which is a tertiary care hospital in northern India catering to local and referred cases from Jammu province. To study burn wound infection, swabs were taken from open burn wounds. Burn wound swabs were taken initially on admission, followed by swabs on day 5th, second, third and fourth week respectively. They were taken before dressing changes and before administration of antibiotics wherever possible. Wound swabs were also taken whenever there were clinical signs of grafted skin infections. The wound swab specimens were inoculated on Blood agar and MacConkey agar and were incubated at $37 \mathrm{C}$ for $24-48$ hours. Identification of bacterial isolates was done using colony morphology, Gram-staining and conventional biochemical tests as per standardized 
protocols of our laboratory. Different panels of antimicrobial agents for Gram-positive and Gram-negative bacteria were used as per Clinical Laboratory Standards Institute (CLSI) guidelines (Bollerao et al., 2003).

\section{Results and Discussion}

A total of 63 patients were enrolled in the present study. Among these, 49 showed evidence of burn wound infection whereas 14 had no evidence of infection. So present study includes only $49(77.7 \%)$ infected patients. Out of 49 patients Females were 27 (55.10\%) and males were 22(44.89\%). The Total Burn Surface Area (TBSA) burned ranged from 5\% to $40 \%$. The types of initial burn insults included flame burns in $21(42.85 \%)$ patients, electrical burns in 17 (34.69\%) patients, and scalds in $11(22.44 \%)$ patients. Gram-positive organisms were predominantly isolated from the burn wounds during the first week of admission ( $S$. aureus being the most frequent isolate from 1st and 5th day of admission wound swabs whereas Gram-negative organisms were common from second week onwards with Pseudomonas sp. being the most common isolate from the 2nd, 3rd and 4th week. Table 1 shows bacterial isolates from burn wound on different days of admission. Gram-positive organisms were more common in first week of admission whereas gram-negative organism were common from second week onwards.

Table.1 Isolates from burn at varying time periods

\begin{tabular}{|l|l|l|l|l|l|l|}
\hline $\begin{array}{l}\text { Organism } \\
\text { isolated }\end{array}$ & $\begin{array}{l}\text { Day of } \\
\text { Admission }\end{array}$ & On $\mathbf{5}^{\text {th }}$ day & $\mathbf{2}^{\text {nd }}$ week & $\mathbf{3}^{\text {rd }}$ week & $\mathbf{4}^{\text {th }}$ week & Total \\
\hline S. aureus & $8(26.66 \%)$ & $09(26.47 \%)$ & 0 & 0 & 0 & $17(17 \%)$ \\
\hline Streptococcus $\boldsymbol{s p}$. & $05(16.66 \%)$ & $05(14.70 \%)$ & 0 & 0 & 0 & $10(10 \%)$ \\
\hline Enterococcus $\boldsymbol{s} \boldsymbol{p}$. & $04(13.33 \%)$ & 0 & 0 & 0 & 0 & $4(4 \%)$ \\
\hline Pseudomonas $\boldsymbol{s p}$. & $06(20 \%)$ & $08(23.52 \%)$ & $07(29.16 \%)$ & $04(50 \%)$ & $02(50 \%)$ & $27(27 \%)$ \\
\hline Klebsiella sp. & $04(13.33 \%)$ & $06(17.64 \%)$ & $11(45.83 \%)$ & $03(37.5 \%)$ & $2(50 \%)$ & $26(26 \%)$ \\
\hline Proteus $\boldsymbol{s p}$. & $03(10 \%)$ & $05(14.7 \%)$ & $03(12.5 \%)$ & 0 & 0 & $11(11 \%)$ \\
\hline Enterobacter $\boldsymbol{s p}$. & 0 & $01(2.94 \%)$ & $02(8.33 \%)$ & $01(12.5 \%)$ & 0 & $04(4 \%)$ \\
\hline Acinetobacter $\boldsymbol{s p}$. & 0 & 0 & $01(4.16 \%)$ & 0 & 0 & $01(1 \%)$ \\
\hline Total & 30 & 34 & 24 & 8 & 4 & 100 \\
\hline
\end{tabular}

Table.2 Antibiotic Susceptibility pattern of Gram-positive organisms

(A) Antibiotic sensitivity pattern on Admission (No. of Sensitive isolates/No. of Total isolates)

\begin{tabular}{|l|l|l|l|l|l|l|l|l|l|l|l|l|l|l|}
\hline Isolates & $\mathbf{P}$ & $\mathbf{C f x}$ & $\mathbf{G}$ & $\mathbf{C f}$ & $\mathbf{C o}$ & $\mathbf{V a}$ & $\mathbf{C d}$ & $\mathbf{E}$ & $\mathbf{L z}$ & $\mathbf{C}$ & $\mathbf{T}$ & $\mathbf{C i}$ & $\mathbf{C p m}$ & $\mathbf{O x}$ \\
\hline S.aureus & $1 / 8$ & $5 / 8$ & $2 / 8$ & $2 / 8$ & $3 / 8$ & $8 / 8$ & $1 / 8$ & $3 / 8$ & $6 / 8$ & $3 / 8$ & $1 / 8$ & 0 & $2 / 8$ & $2 / 8$ \\
\hline $\begin{array}{l}\text { Staphylo } \\
\text { coccus sp. }\end{array}$ & $1 / 5$ & $3 / 5$ & 0 & 0 & $2 / 5$ & $5 / 5$ & 0 & $1 / 5$ & $3 / 5$ & 0 & 0 & $1 / 5$ & $2 / 5$ & $1 / 5$ \\
\hline $\begin{array}{l}\text { Entero } \\
\text { coccus sp. }\end{array}$ & 0 & 0 & 0 & $3 / 4$ & 0 & $4 / 4$ & 0 & $1 / 4$ & 0 & $1 / 4$ & 0 & 0 & 0 & 0 \\
\hline
\end{tabular}

Abbreviations: P-Penicillin, Cfx-Cefoxitin, G-Gentamycin, Cf-Ciprofloxacin, Co-Cotrimoxazole, Va-Vancomycin, Cd-Clindamycin, E-Erythromycin, Lz-Linezolid, C-Chloramphenicol, T-Tetracycline, Ci-Ceftrioxone, CpmCefipime, Ox-Oxacillin. 
(B) Antibiotic sensitivity pattern on Day 5

\begin{tabular}{|l|l|l|l|l|l|l|l|l|l|l|l|l|l|l|}
\hline Isolates & $\mathbf{P}$ & $\mathbf{C f x}$ & $\mathbf{G}$ & $\mathbf{C f}$ & $\mathbf{C o}$ & $\mathbf{V a}$ & $\mathbf{C d}$ & $\mathbf{E}$ & $\mathbf{L z}$ & $\mathbf{C}$ & $\mathbf{T}$ & $\mathbf{C i}$ & $\mathbf{C p m}$ & $\mathbf{O x}$ \\
\hline S. aureus & $1 / 9$ & $4 / 9$ & $2 / 9$ & $3 / 9$ & $3 / 9$ & $9 / 9$ & $2 / 9$ & $2 / 9$ & $5 / 9$ & $4 / 9$ & $2 / 9$ & 0 & $3 / 9$ & $3 / 9$ \\
\hline $\begin{array}{l}\text { Staphyloco } \\
\text { ccus sp. }\end{array}$ & $1 / 5$ & $2 / 4$ & 0 & 0 & $3 / 4$ & $5 / 5$ & 0 & $1 / 4$ & $3 / 4$ & 0 & 0 & $1 / 4$ & $2 / 4$ & $2 / 4$ \\
\hline $\begin{array}{l}\text { Enterococ } \\
\text { cus sp. }\end{array}$ & 0 & 0 & 0 & 0 & 0 & 0 & 0 & 0 & 0 & 0 & 0 & 0 & 0 & 0 \\
\hline
\end{tabular}

Abbreviations: P-Penicillin, Cfx-Cefoxitin, G-Gentamycin, Cf-Ciprofloxacin, Co-Cotrimoxazole, Va-Vancomycin, Cd-Clindamycin, E-Erythromycin, Lz-Linezolid, C-Chloramphenicol, T-Tetracycline, Ci-Ceftrioxone, CpmCefipime, Ox-Oxacillin.

Table.3 Antibiotic Susceptibility pattern of Gram-negative organisms

(A) Antibiotic sensitivity pattern on Admission (No. of Sensitive isolates/No. of Total isolates)

\begin{tabular}{|l|l|l|l|l|l|l|l|l|l|l|l|l|l|l|l|l|l|}
\hline Isolates & $\mathbf{A}$ & $\mathbf{P t}$ & $\mathbf{C a}$ & $\mathbf{C p m}$ & $\mathbf{C i}$ & $\mathbf{C u}$ & $\mathbf{A k}$ & $\mathbf{I}$ & $\mathbf{G}$ & $\mathbf{T b}$ & $\mathbf{C f}$ & $\mathbf{C o}$ & $\mathbf{C}$ & $\mathbf{T}$ & $\mathbf{C l}$ & $\mathbf{P b}$ & $\mathbf{C s}$ \\
\hline Pseudomonas sp. & 0 & $5 / 6$ & $3 / 6$ & 0 & 0 & 0 & 0 & 0 & 0 & 0 & 0 & 0 & 0 & 0 & 0 & $3 / 6$ & 0 \\
\hline Klebsiella sp. & 0 & $4 / 4$ & $4 / 4$ & 0 & 0 & 0 & $2 / 4$ & $2 / 4$ & 0 & 0 & 0 & 0 & 0 & 0 & 0 & 0 & 0 \\
\hline Proteus sp. & 0 & $3 / 3$ & 0 & $1 / 3$ & 0 & 0 & 0 & $1 / 3$ & 0 & 0 & 0 & 0 & 0 & 0 & 0 & 0 & 0 \\
\hline Enterobacter sp. & 0 & 0 & 0 & 0 & 0 & 0 & 0 & 0 & 0 & 0 & 0 & 0 & 0 & 0 & 0 & 0 & 0 \\
\hline Acinetobacter sp. & 0 & 0 & 0 & 0 & 0 & 0 & 0 & 0 & 0 & 0 & 0 & 0 & 0 & 0 & 0 & 0 & 0 \\
\hline
\end{tabular}

Abbreviation: A-Ampicillin, Pt-Piperacillin tazobactum, Ca-Ceftazidime, Cpm-Cefipime, Ci-Ceftrioxone, CuCefuroxime, Ak-Amikacin, I-Imipenem, G-Gentamycin, Tb-Tobramycin, Cf-Ciprofloxacin, Co-Cotrimoxazole, CChloramphenicol, T-Tetracycline, Cl-Colistin, $\mathrm{Pb}$-Polymyxin B, Cs-Cefoperazonesulbactum.

(B) Antibiotic sensitivity pattern on Day 5

\begin{tabular}{|l|l|l|l|l|l|l|l|l|l|l|l|l|l|l|l|l|l|}
\hline Isolates & $\mathbf{A}$ & $\mathbf{P t}$ & $\mathbf{C a}$ & $\mathbf{C p m}$ & $\mathbf{C i}$ & $\mathbf{C u}$ & $\mathbf{A k}$ & $\mathbf{I}$ & $\mathbf{G}$ & $\mathbf{T b}$ & $\mathbf{C f}$ & $\mathbf{C o}$ & $\mathbf{C}$ & $\mathbf{T}$ & $\mathbf{C l}$ & $\mathbf{P b}$ & $\mathbf{C s}$ \\
\hline Pseudomonas $\boldsymbol{s p}$. & 0 & $7 / 8$ & $7 / 8$ & $4 / 8$ & $2 / 8$ & 0 & 0 & $2 / 8$ & 0 & 0 & 0 & 0 & 0 & 0 & $2 / 8$ & $7 / 8$ & 0 \\
\hline Klebsiella $\boldsymbol{s p}$. & $1 / 6$ & $4 / 4$ & $4 / 6$ & 0 & 0 & 0 & $2 / 6$ & $4 / 6$ & $3 / 6$ & $2 / 6$ & 0 & 0 & 0 & 0 & 0 & 0 & 0 \\
\hline Proteus sp. & 0 & $4 / 5$ & $2 / 5$ & $4 / 5$ & 0 & 0 & 0 & $2 / 5$ & 0 & 0 & 0 & 0 & 0 & 0 & 0 & $2 / 5$ & 0 \\
\hline Enterobacter $\boldsymbol{s p}$. & 0 & $1 / 1$ & $1 / 1$ & $1 / 1$ & 0 & 0 & 0 & 0 & 0 & 0 & 0 & 0 & 0 & 0 & 0 & 0 & 0 \\
\hline Acinetobacter $\boldsymbol{s p}$. & 0 & 0 & 0 & 0 & 0 & 0 & 0 & 0 & 0 & 0 & 0 & 0 & 0 & 0 & 0 & 0 & 0 \\
\hline
\end{tabular}

Abbreviation: A-Ampicillin, Pt-Piperacillin tazobactum, Ca-Ceftazidime, Cpm-Cefipime, Ci-Ceftrioxone, Cu-

Cefuroxime, Ak-Amikacin, I-Imipenem, G-Gentamycin, Tb-Tobramycin, Cf-Ciprofloxacin, Co-Cotrimoxazole, C-

Chloramphenicol, T-Tetracycline, Cl-Colistin, $\mathrm{Pb}$-Polymyxin B, Cs-Cefoperazonesulbactum

(C) Antibiotic sensitivity pattern on Week 2

\begin{tabular}{|l|l|l|l|l|l|l|l|l|l|l|l|l|l|l|l|l|l|}
\hline Isolates & $\mathbf{A}$ & $\mathbf{P t}$ & $\mathbf{C a}$ & $\mathbf{C p m}$ & $\mathbf{C i}$ & $\mathbf{C u}$ & $\mathbf{A k}$ & $\mathbf{I}$ & $\mathbf{G}$ & $\mathbf{T b}$ & $\mathbf{C f}$ & $\mathbf{C o}$ & $\mathbf{C}$ & $\mathbf{T}$ & $\mathbf{C l}$ & $\mathbf{P b}$ & $\mathbf{C s}$ \\
\hline $\begin{array}{l}\text { Pseudomonas } \\
\text { sp. }\end{array}$ & 0 & $6 / 7$ & $3 / 7$ & $4 / 7$ & 0 & 0 & $1 / 7$ & 0 & 0 & 0 & 0 & 0 & 0 & 0 & 0 & 0 & $4 / 7$ \\
\hline Klebsiella sp. & $1 / 11$ & $11 / 11$ & $6 / 11$ & $7 / 11$ & 0 & $2 / 11$ & $7 / 11$ & $8 / 11$ & $5 / 11$ & $3 / 11$ & 0 & 0 & 0 & 0 & 0 & 0 & 0 \\
\hline Proteus sp. & 0 & $3 / 3$ & $2 / 3$ & $2 / 3$ & 0 & 0 & 0 & 0 & 0 & 0 & 0 & 0 & 0 & 0 & 0 & 0 & $1 / 3$ \\
\hline Enterobacter $\boldsymbol{s}$. & 0 & $2 / 2$ & $1 / 2$ & $1 / 2$ & 0 & 0 & $1 / 2$ & $2 / 2$ & $1 / 2$ & 0 & 0 & 0 & 0 & 0 & 0 & 0 & 0 \\
\hline $\begin{array}{l}\text { Acinetobacter } \\
\text { sp. }\end{array}$ & 0 & $1 / 1$ & 0 & 0 & 0 & 0 & 0 & 0 & 0 & 0 & 0 & $1 / 1$ & 0 & 0 & 0 & 0 & 0 \\
\hline
\end{tabular}

Abbreviation: A-Ampicillin, Pt-Piperacillin tazobactum, Ca-Ceftazidime, Cpm-Cefipime, Ci-Ceftrioxone, Cu-

Cefuroxime, Ak-Amikacin, I-Imipenem, G-Gentamycin, Tb-Tobramycin, Cf-Ciprofloxacin, Co-Cotrimoxazole, C-

Chloramphenicol, T-Tetracycline, Cl-Colistin, $\mathrm{Pb}$-Polymyxin B, Cs-Cefoperazonesulbactum 
(D)Antibiotic sensitivity pattern on Week 3

\begin{tabular}{|c|c|c|c|c|c|c|c|c|c|c|c|c|c|c|c|c|c|}
\hline Isolates & $\mathbf{A}$ & $\mathbf{P t}$ & $\begin{array}{l}\mathbf{C} \\
\mathbf{a}\end{array}$ & $\begin{array}{l}\mathbf{C p} \\
\mathbf{m}\end{array}$ & $\mathbf{C i}$ & $\begin{array}{l}\mathbf{C} \\
\mathbf{u}\end{array}$ & $\begin{array}{l}\mathbf{A} \\
\mathbf{k} \\
\end{array}$ & I & G & $\begin{array}{l}\text { T } \\
\text { b }\end{array}$ & $\begin{array}{l}\text { C } \\
\text { f }\end{array}$ & $\begin{array}{l}\mathrm{C} \\
\mathbf{0} \\
\end{array}$ & C & $\mathbf{T}$ & $\mathrm{Cl}$ & $\begin{array}{l}\mathbf{P} \\
\mathbf{b} \\
\end{array}$ & \\
\hline $\begin{array}{l}\text { Pseudom } \\
\text { onas sp. }\end{array}$ & 0 & $3 / 4$ & $\begin{array}{l}2 / \\
4\end{array}$ & $1 / 4$ & (0) & (0 & 0 & 0 & 0 & 0 & 0 & $\mathbf{0}$ & 0 & 0 & (0) & $\begin{array}{l}3 / \\
4\end{array}$ & $\mathbf{0}$ \\
\hline $\begin{array}{l}\text { Klebsiell } \\
\text { a sp. }\end{array}$ & 0 & $3 / 3$ & $\begin{array}{l}2 / \\
3\end{array}$ & $2 / 3$ & $\mathbf{0}$ & $\begin{array}{l}1 / \\
3\end{array}$ & $\begin{array}{l}2 / \\
3\end{array}$ & $\begin{array}{l}2 / \\
3\end{array}$ & $\begin{array}{l}1 / \\
3\end{array}$ & $\begin{array}{l}1 / \\
3\end{array}$ & 0 & $\mathbf{0}$ & 0 & $\mathbf{0}$ & (0 & 0 & $\mathbf{0}$ \\
\hline $\begin{array}{l}\text { Proteus } \\
\text { sp. }\end{array}$ & 0 & 0 & 0 & 0 & 0 & 0 & 0 & 0 & 0 & 0 & 0 & 0 & 0 & 0 & 0 & 0 & $\mathbf{0}$ \\
\hline $\begin{array}{l}\text { Enterob } \\
\text { acter } s p .\end{array}$ & 0 & $1 / 1$ & 0 & $1 / 1$ & 0 & 0 & 0 & $\begin{array}{l}1 / \\
1\end{array}$ & 0 & 0 & 0 & $\mathbf{0}$ & 0 & (0 & (0) & 0 & $\mathbf{0}$ \\
\hline $\begin{array}{l}\text { Acinetob } \\
\text { acter sp. }\end{array}$ & 0 & 0 & 0 & 0 & 0 & $\mathbf{0}$ & $\mathbf{0}$ & 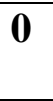 & $\mathbf{0}$ & $\mathbf{0}$ & 0 & $\mathbf{0}$ & 0 & O & 0 & 0 & $\mathbf{0}$ \\
\hline
\end{tabular}

Abbreviation: A-Ampicillin, Pt-Piperacillin tazobactum, Ca-Ceftazidime, Cpm-Cefipime, Ci-Ceftrioxone, CuCefuroxime, Ak-Amikacin, I-Imipenem, G-Gentamycin, Tb-Tobramycin, Cf-Ciprofloxacin, Co-Cotrimoxazole, CChloramphenicol, T-Tetracycline, Cl-Colistin, $\mathrm{Pb}$-Polymyxin B, Cs-Cefoperazonesulbactum

(E) Antibiotic sensitivity pattern on Week 4

\begin{tabular}{|c|c|c|c|c|c|c|c|c|c|c|c|c|c|c|c|c|c|}
\hline Isolates & $\mathbf{A}$ & $\mathbf{P t}$ & $\begin{array}{l}\text { C } \\
\text { a }\end{array}$ & $\begin{array}{l}\text { Cp } \\
\mathbf{m}\end{array}$ & $\mathrm{Ci}$ & $\begin{array}{l}\mathbf{C} \\
\mathbf{u}\end{array}$ & $\begin{array}{l}\mathbf{A} \\
\mathbf{k}\end{array}$ & I & C & $\begin{array}{l}\text { T } \\
\text { b }\end{array}$ & c & $\begin{array}{l}\mathbf{C} \\
\mathbf{0}\end{array}$ & C & $\mathbf{T}$ & Cl & $\begin{array}{l}\mathbf{P} \\
\text { b }\end{array}$ & \begin{tabular}{|l|}
$\mathbf{C}$ \\
$\mathbf{s}$ \\
\end{tabular} \\
\hline $\begin{array}{l}\text { Pseudom } \\
\text { onas sp. }\end{array}$ & 0 & $2 / 2$ & 0 & $1 / 2$ & 0 & 0 & 0 & $\overline{11}$ & 0 & 0 & 0 & 0 & 0 & 0 & 0 & $\begin{array}{l}3 / \\
4 \\
\end{array}$ & \begin{tabular}{|l|l|}
0 \\
\end{tabular} \\
\hline $\begin{array}{l}\text { Klebsiella } \\
\text { sp. }\end{array}$ & 0 & $2 / 2$ & 0 & $1 / 2$ & (0 & 0 & 0 & $1 /$ & $\begin{array}{l}1 \\
2\end{array}$ & $\mathbf{0}$ & $\mathbf{0}$ & 0 & 0 & $\mathbf{0}$ & $\mathbf{0}$ & $\mathbf{0}$ & \begin{tabular}{|l|l}
0 \\
\end{tabular} \\
\hline $\begin{array}{l}\text { Proteus } \\
\text { sp. }\end{array}$ & $\overline{\mathbf{0}}$ & 0 & $\overline{\mathbf{0}}$ & $\overline{0}$ & $\overline{\mathbf{0}}$ & O & O & 0 & 0 & $\mathbf{0}$ & 0 & 0 & 0 & $\mathbf{0}$ & $\mathbf{0}$ & $\mathbf{0}$ & $\mathbf{0}$ \\
\hline $\begin{array}{l}\text { Enteroba } \\
\text { cter sp. }\end{array}$ & 0 & 0 & 0 & (0 & 0 & 0 & 0 & 0 & 0 & 0 & 0 & 0 & 0 & $\mathbf{0}$ & 0 & 0 & \begin{tabular}{|l|l}
$\mathbf{0}$ \\
\end{tabular} \\
\hline $\begin{array}{l}\text { Acinetob } \\
\text { acter } \mathrm{sp} .\end{array}$ & 0 & 0 & 0 & 0 & 0 & 0 & 0 & 0 & 0 & 0 & 0 & 0 & 0 & $\mathbf{0}$ & 0 & 0 & $\begin{array}{ll}0 \\
\end{array}$ \\
\hline
\end{tabular}

Abbreviation: A-Ampicillin, Pt-Piperacillin tazobactum, Ca-Ceftazidime, Cpm-Cefipime, Ci-Ceftrioxone, CuCefuroxime, Ak-Amikacin, I-Imipenem, G-Gentamycin, Tb-Tobramycin, Cf-Ciprofloxacin, Co-Cotrimoxazole, CChloramphenicol, T-Tetracycline, Cl-Colistin, $\mathrm{Pb}$-Polymyxin B, Cs-Cefoperazonesulbactum.

Fresh burn is usually sterile but progressively becomes colonized with one or more bacterial species. The role of different bacterial species in burn pathology varies from mere colonization, local tissue sepsis, interference with healing and grafting, to invasion of the blood stream with subsequent septicaemia and death (Hansbrough et al., 1987). The burn is considered one of the major health problems in the world, and infection is one of the most frequent and severest of complications in these patients. The standard techniques for microbiological detection remain surface swabbing and wound biopsy culture; having its advocates and its critics. The surface swab probably remains the work horse. It is relatively inexpensive technique that most commonly is used to provide qualitative information about the bacteria present (Lederer et al., 1999). 
The present study conducted to isolate and identify different bacteria from burn wound infections, to check the pattern of changes in flora with time and to determine their antimicrobial sensitivity in the Department of Microbiology, Government Medical College Hospital, and Jammu. A total of 63 patients were enrolled in study out of which $49(77.77 \%)$ showed evidence of burn wound infection whereas $14(22.22 \%)$ had no microbiological and clinical evidence of infection. Gram positive bacteria predominantly isolated from the burn wounds during the first week of admission ( $S$. aureus being the most frequent isolate from 1 st and 5th day of admission wound swabs whereas Gram-negative organisms were common from second week onwards with pseudomonas $s p$. being the most common isolate from the 2 nd, 3rd and 4th week. A study conducted by Manjula et al., (2007) which reported that Pseudomonas species was the commonest gram negative bacteria isolated (23.33\%) from burn wounds followed by gram positive bacteria (Staphylococcus aureus) which is in agreement with findings of our study.(15) Our results showed that the rate of isolation of gram-negative organism was more than grampositive, these results are consistent with those reported by Kehinde et al., (2004) who reported that the rate of gram negative bacterial isolation from burn wound was more than twice that gram- positive. In our study the results of antimicrobial sensitivity showed Vancomycin was $100 \%$ sensitive drug to Gram Positive bacteria (S. aureus, Staphylococcus sp. Enterococcus sp.) Gramnegative organisms isolated at the time of admission were highly resistant to most of first, second and third line antibiotics. Piperacillin-tazobactum was most susceptible drugs for gram negative bacteria. Klebsiella sp., Enterobacter sp. and Acinetobacter showed $100 \%$ sensitivity to Piperacillintazobactum. According to the study conducted by Saxena et al., (2013) high level of drug resistance was observed for cefotaxime, ceftazidime, and cotrimoxazole among Gram-negative pathogens. Piperacillin/tazobactam, Imipenem, Amikacin, and Ciprofloxacin were found to be the sensitive drug which is in concordant with the findings of the present study (Saxena et al., 2013). The pattern of bacterial resistance is important for epidemiological and clinical purposes. The results of the antimicrobial resistance pattern give serious cause for concern because the predominant bacterial isolates were highly resistant to the commonly available antimicrobial agents.

Conclusion Gram-positive organisms predominated as colonizing agents of the burn wounds initially but later it was dominated by gram-negative bacteria. The most common infectious organisms in the burn unit were $S$. aureus and pseudomonas $\mathrm{s} p$. The isolates showed a moderate degree of resistance to the commonly used antibiotics. The design and discipline in the unit can be considered satisfactory in managing the burn population However, as patients with longer duration of injury are admitted, there is always a possibility of introduction of infected wounds (including infections with MDR organisms) into the burn unit. There is a need for action surveillance and monitoring of the burns wards and personnel in order to decrease the infection rate in future.

\section{References}

American Burn Association. 2000. Burn incidence and treatment in the US: 2000 fact sheet. http://www.ameriburn.org.

Apelgren, P., Bjornhagen, V., Bragderyd, K., Jonsson, C.E., Ransjo, U. 2002. A prospective study of infections in burn patients. Burns, 28(1): 39-46.

Atiyeh, B.S., S.W. Gunn, and S.N. Hayek. 2005. State of the art in burn Treatment. World J. Surg., 29: 131-148. 
Baker, C.C., C.L. Miller, and D.D. Trunkey. 1979. Predicting fatal sepsis in burn patients. J. Trauma, 19: 641-648.

Bollerao, D., Cortellini, M., Stella, M., Carnino, R., Magliacani, G., Iorio, M. 2003. Pan-Antibiotic resistance and nosocomial infection in burn patients: therapeutic choices and legal problems in Italy. Ann. Burns and Fire Dis., 16(4).

Challa Negri. Microbiology of Burn Unit at Yekatit 12 Hospital, Addis Ababa.

Hansbrough, J.F., T.O. Field, Jr., M.A. Gadd, and C. Soderberg. 1987. Immune response modulation after burn injury: T cells and antibodies. J. Burn Care Rehabil., 8: 509-512.

Karyoute, S. 1989. Burn wound infection in 100 patients treated in the burn unit at Jordan University Hospital. Burns, 15(1): 117-119

Kehinde, A.O., Ademola, S.A., Okesola, A.O. and Oluwatosin, O.M. 2004. Pattern of bacterial pathogens in burn wound infections in Ibadan, Nigeria. Annals of Burns and fire disasters, Vol XVII: 348-355.

Lederer, J.A., M.L. Rodrick, and J.A. Mannick. 1999. The effects of injury on the adaptive immune response. Shock, 11: 153-159.

Lemmen, S., Hafner, H., Zolldann, D., Stanzel, S., Lutticken, R. 2004.
Distribution of multi-resistant gram negative versus gram-positive bacteria in the hospital inanimate environment Burns, 56: 191-197.

Lionelli, G.T., E.J. Pickus, O.K. Beckum, R.L. Decoursey, and R.A. Korentager. 2005. A three decade analysis of factors affecting burn mortality in the elderly. Burns, 31: 958-963.

Manjula, M., Priya, D. and Varsha, G. 2007. Bacterial isolates from burn wound infections and antibiograms: eight year study. Ind. J. Plastic Surg., 40: 22-25.

Neelam, T., Rekha, E., Chari, P., Meera, S. 2004. A prospective study of hospitalacquired infections in burn patients at a referral centre in North India. Burns, 30: 665-669.

Pruitt, B., McManus, A., Kim, S., Goodwin, C. 1998. Burn wound infections: current status. World J. Surg., 22(2): 135-145.

Saxena, N., Dadhich, D., Maheshwari, D. 2013. Aerobic bacterial isolates from burn wound infection patients and their antimicrobial susceptibility pattern in Kota, Rajasthan. J. Evol. Med. Dent. Sci., 2: 4156-60.

Zorgani, A., Zaidi, M., Franka, R., Shahen, A. 2002. The pattern and outcome of septicaemia in a burns intensive care unit. Annals of Burns and Fire Disasters, 15(4).

\section{How to cite this article:}

Shashi S. Sudhan, Preeti Sharma, Kunal Sharma, Monika Sharma and Sorabh Singh Sambyal. 2017. Time Related Emergence of Bacterial Pathogens and their Antibiograms in Burn Wound Infections in a Tertiary Care Hospital. Int.J.Curr.Microbiol.App.Sci. 6(4): 416-422. doi: https://doi.org/10.20546/ijcmas.2017.604.047 